\title{
A Novel Adaptive Distance Protection Principle for Distribution System with Distributed Generators
}

\author{
Jianfang $\mathrm{Li}^{1, \text { a }}$, Xiaohui Song ${ }^{1, \mathrm{~b}}$ and Jing $\mathrm{Ma}^{2, \mathrm{c}}$ \\ ${ }^{1}$ Power Distribution Department, China Electric Power Research Institute, Beijing, 100192, China \\ 2 State Key Laboratory of Alternate Electrical Power System with Renewable Energy Sources, \\ North China Electric Power University, Beijing, 102206, China \\ ahdmajing@163.com, bhdmajing@ncepu.edu.cn, 'hamajing@aliyun.com.cn
}

\begin{abstract}
Keywords: Power system, Distributed generation (DG), Distribution network, Adaptive distance protection
\end{abstract}

\begin{abstract}
The value setting of the traditional distance protection has become very difficult for the distribution system with distributed generators (DGs) owing to the randomness of the DG output power. This paper proposes a new adaptive distance protection scheme to solve this problem. Firstly, on the basis of the connected point of DGs, the protected feeders are divided into different zones, with adaptive distance protection configured at the upstream side of DG. Then, based on a fault characteristic analysis of the distribution system with $\mathrm{DG}$, the adaptive distance protection scheme for the distribution system with DG is studied to solve this problem. The results show that this scheme can automatically calculate the settings according to the operation mode and the output power of DG with no need for communication. Compared with the traditional adaptive protection scheme, the performance of this method is greatly improved. Simulation results on a $10 \mathrm{kV}$ distribution system have verified the validity of the scheme proposed.
\end{abstract}

\section{Introduction}

Distributed generation (DG) is by definition generation which is of limited size (few kilowatts to few megawatts) and interconnected at substation, distribution feeder or customer load level [1-5]. DG technologies include photovoltaics, wind turbines, fuel cells, micro turbines, gas turbines and internal combustion engines [2-3, 5]. DGs can be divided into two categories: traditional rotating machine DG and inverter interfaced DG (IIDG). Rotating machine DG can be equivalent to series connection of voltage source and impedance, whereas IIDG is connected to power system via power electronic devices [6-7]. Even wind power and micro gas turbine are also connected to power grid via rectifying-inverting process, to avoid the problems brought by direct connection of traditional generator. IIDG usually adopts DC-type PQ-mode control, namely makes DG output P and $\mathrm{Q}$ achieve the setting value by adjusting output current of the inverter.

The cost of transmission and distribution is rising, but the costs of DG technologies are falling. This makes it more economical to meet an increase of load by connecting DGs to distribution feeders rather than expanding transmission and distribution (T\&D) facilities [4]. Therefore, these technologies are entering a period of rapid expansion and commercialization and studies have predicted that DGs may account up to $20 \%$ of all new generation going on line by year 2011 [1]. In such a system, DGs would feed loads around its location, thus relieving the burden on the source. This clearly suggests that the basic assumption of distribution system being radial is not likely to hold in near future. One would then be looking at a multisource unbalanced system. It is a wellestablished fact that protection devices in a multisource system have to be direction sensitive [8-10]. Hadjsaid et al. [11] show through a simple example that fault currents through protective devices would change after introduction of DG. They further suggest checking protection selectivity and sensitivity for each new connection of DG. Thus the study of adaptive protection for distributed system with DGs is demonstrated in this paper. The protected feeders are firstly divided into different zones, with adaptive distance protection configured at the upstream side of DG. Then, the 
adaptive distance protection scheme for the distribution system with DG is proposed. Simulation results on a $10 \mathrm{kV}$ distribution system have verified that the scheme is able to clear the fault with high sensitivity and selectivity.

\section{Impact of DG on Protection Coordination}

A typical case is shown in Fig. 1. An industrial power network is fed through source $G$ and protected by R1, R2, and R3. Each protective device is assigned a primary function to clear faults in a specific zone and a secondary function to clear faults in the adjacent or downstream zones to the extent within the range of the device permits. In this situation, the next upstream device, or device combination, must operate to provide backup protection. When two devices operate properly in this primary/secondary mode for any system fault, they are said to be coordinated. Proper coordination is achieved by this discrimination between successive devices. Good practice dictates that when a fault F1 occurs, the time of operation of relay R2 should be made larger than the time of operation of R1 at least by a time interval called the "coordination time interval." As clearly shown in Fig. 1, R2 will back up R1.

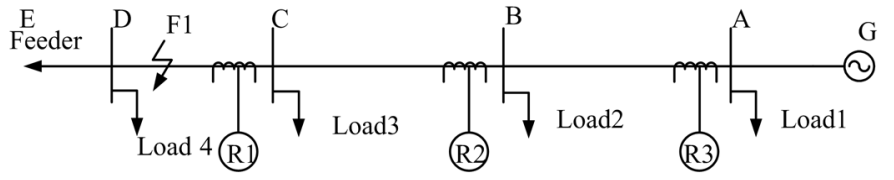

Fig.1. An industrial power network fed through source $\mathrm{G}$ and protected by R1, R2, and R3.

It is clear that protection for distribution systems with DG cannot be achieved with the same philosophies that have been used to protect traditional distribution systems. At the very least, a system designed to protect distribution systems with DG should take the following into consideration.

1) Bidirectional Current Flow: DG1 and DG2 connect to the system as shown in Fig. 2. For F2, selectivity requires that R2 operates before R1, and for F1, R1 should operate before R2. Therefore, the system should be facilitated with two directional protection devices at each line to ensure the correct fault isolation, as shown in Fig. 3.

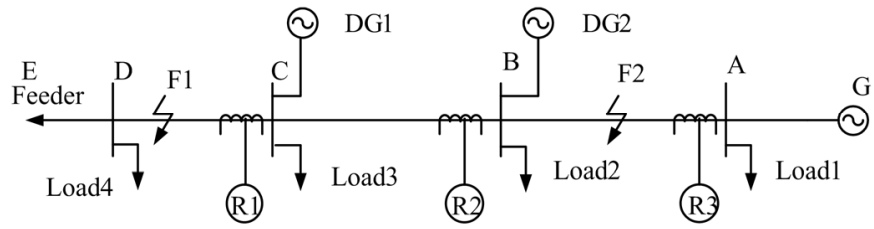

Fig.2. An industrial power network fed through source G, DG1, and DG2, and protected by R1, R2, and R3.

2) Changing System Conditions: For F1, The fault current now has two components-one coming from the supply, and the other from DG1 and DG2. Under the condition of changing of capacities of DGs, the maximum and minimum fault currents will change. This will require R1 and R2 to be coordinated at changing current conditions.

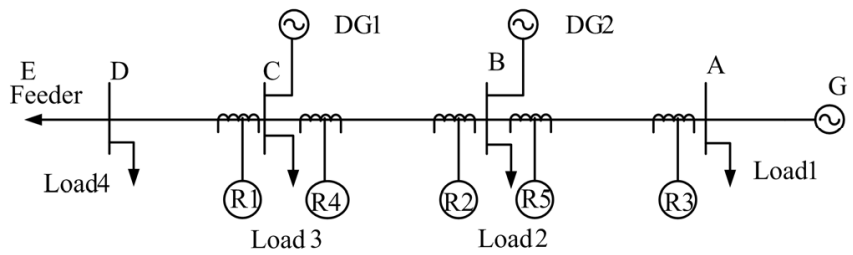

Fig.3. An industrial power network with directional protection devices to ensure the correct fault isolation.

In addition, due to the size and placement of DGs, some potential cases are also not well coordinated in an actual distribution system [6]. The only way to withhold coordination in presence of DG penetration is to throw off all DGs when fault occurs. However, this would cause DG disconnected for temporary faults.

\section{Adaptive Scheme}

In the transient process of fault, the output power will increase in short time [12]. Then the IIDG 
output $\mathrm{P}$ and $\mathrm{Q}$ return to the setting value very quickly, whereas the output current becomes larger than the pre-fault value due to voltage reduction. Furthermore, the three-phase output currents of IIDG are always equal during the transient process. Thus, IIDG only provides positive sequence current no matter what type of fault occurs. Zone I of distance protection is not affected by the IIDG and can be calculated by conventional method. However, Zone II should coordinate with Zone I of distance protection of next line [13]-[14]. Therefore, this paper mainly focuses on value setting of Zone II of distance protection.

Distribution system containing an IIDG is shown in Fig. 4. Relay 2 has an infeed current provided by IIDG. For three-phase fault, the setting value of Zone II of relay 2 is given by:

$$
Z_{\text {set. } 2}^{\mathrm{II}}=K_{\text {rel }}^{\mathrm{II}}\left(Z_{B C}+K_{b} Z_{\text {set. } 3}^{\mathrm{I}}\right) \text {. }
$$

where $K_{\mathrm{b}}$ is the branch coefficient. $K_{\mathrm{b}}$ is calculated as:

$$
K_{b}=1+\frac{\dot{I}_{D G}}{\dot{I}_{2}} .
$$

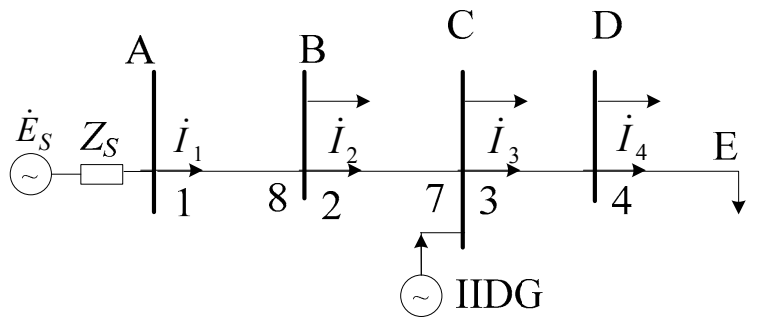

Fig. 4. Schematic diagram of power system containing IIDG.

For the two-phase short circuit fault, branch coefficient can not be calculated by (2) directly. If $B-C$ phase fault occurs at the end of zone I of relay 3, currents at relay 2 and relay 3 is calculated as:

$$
\begin{aligned}
& \left\{\begin{array}{l}
\dot{I}_{2 . b}^{(1)}=\dot{I}_{3 . b}^{(1)}-\dot{I}_{D G . b} \\
\dot{I}_{2 . c}^{(1)}=\dot{I}_{3 . c}^{(1)}-\dot{I}_{D G . c}
\end{array} .\right. \\
& \left\{\begin{array}{l}
\dot{I}_{2 . b}^{(2)}=\dot{I}_{3 . b}^{(2)} \\
\dot{I}_{2 . c}^{(2)}=\dot{I}_{3 . c}^{(2)}
\end{array} .\right.
\end{aligned}
$$

where $\dot{I}_{2 . b}^{(1)}$ is positive sequence current of phase $B$ at relay $2, \dot{I}_{2 . b}^{(2)}$ is negative sequence current, $\dot{I}_{2 . c}^{(1)}$ is positive sequence current of phase $B$ at relay $2, \dot{I}_{2 . c}^{(2)}$ is negative sequence current; $\dot{I}_{3 . b}^{(1)}$ is positive sequence current of phase $B$ at relay $3, \dot{I}_{3 . b}^{(2)}$ is negative sequence current, $\dot{I}_{3 . c}^{(1)}$ is positive sequence current of phase $C$ at relay $3, \dot{I}_{3 . c}^{(2)}$ is negative sequence current, $\dot{I}_{D G . b}$ is phase $B$ current of IIDG, $\dot{I}_{D G . c}$ is phase $C$ current of IIDG.

Voltages of phase $B$ and phase $C$ at relay 2 are given by:

$$
\left\{\begin{array}{l}
\dot{U}_{2 . b}=\dot{U}_{F . b}+I_{3 . b} Z_{C F}+\left(I_{3 . b}^{(1)}-\dot{I}_{D G . b}\right) Z_{B C}+I_{2 . b}^{(2)} Z_{B C} \\
\dot{U}_{2 . c}=\dot{U}_{F . c}+I_{3 . c} Z_{C F}+\left(I_{3 . c}^{(1)}-\dot{I}_{D G . c}\right) Z_{B C}+I_{2 . c}^{(2)} Z_{B C}
\end{array} .\right.
$$

where $\dot{U}_{2 . b}$ and $\dot{U}_{2 . c}$ are voltages of phase $B$ and phase $C$ at relay 2 , respectively. $\dot{U}_{F . b}$ and $\dot{U}_{F . c}$ are voltages of phase $B$ and phase $C$ at fault point, respectively. $Z_{C F}$ is the positive sequence impedance of the line between relay 3 and fault point, $Z_{B C}$ is positive sequence impedance of the line between relay 2 and relay 3 .

Measured impedance of protection 2 is calculated as:

$$
Z_{2 m}=\frac{\dot{U}_{2 b}-\dot{U}_{2 c}}{\dot{I}_{2 b}-\dot{I}_{2 c}}=Z_{B C}+K_{b} Z_{C F} .
$$

where $K_{b}$ is the branch coefficient, and it can be expressed as:

$$
K_{b}=1+\frac{\dot{I}_{D G . b}-\dot{I}_{D G . c}}{\dot{I}_{2 . b}-\dot{I}_{2 . c}} \text {. }
$$

Substituting (7) into (1), setting value of zone II of relay 2 during two-phase fault can be obtained. 


\section{Testing Results and Analysis}

A $10 \mathrm{kV}$ distribution system [15] in Tianjin power network is used to demonstrate the effectiveness of the adaptive setting method. The structure of the system is shown in Fig. 5. The base capacity is 500MVA, and base voltage is $10.5 \mathrm{kV}$. Branch $\mathrm{AB}, \mathrm{BC}$ and $\mathrm{AF}$ are overhead lines. The parameters of these lines are: $r_{1}=0.27 \Omega / \mathrm{km}, x_{1}=0.347 \Omega / \mathrm{km}$. Branch CD, DE and FG are underground cables. The parameters of these lines are: $r_{1}=0.259 \Omega / \mathrm{km}, x_{1}=0.093 \Omega / \mathrm{km}$. Each load are connected to each bus with the nominal capacity 6MVA and nominal power factor 0.85 . IIDG with $\mathrm{P}-\mathrm{Q}$ control mode is connected to bus $\mathrm{C}$ and its nominal capacity is 10MVA. The simulation is carried out on PSCAD/EMTDC.

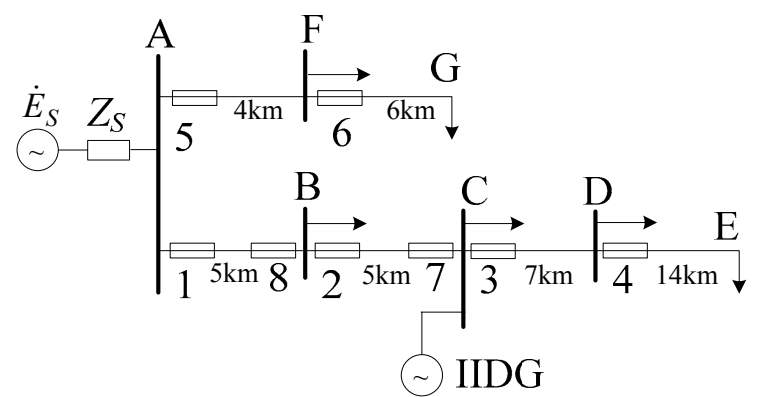

Fig. 5. Structure of example power system in Tianjin.

\subsection{Analysis of Zone II Protection}

Integration of IIDG makes the area between buses $\mathrm{A}$ and $\mathrm{C}$ a double-terminal power system, thus relays 7 and 8 are added to clear fault between buses $\mathrm{A}$ and $\mathrm{C}$. Zone I of relays 1 to 8 is not affected by the IIDG, and can be calculated by conventional method. However, Zone II of relays 2 and 7 are affected by infeed branch coefficient of generator current. Setting of relay 2 is carried out with the method motioned above. Considering infeed current of the traditional power source $\dot{E}_{S}$, setting value of relay 8 is calculated as:

$$
Z_{\text {set. } .8}^{\mathrm{II}}=K_{\text {rel }}^{\mathrm{II}}\left(Z_{B A}+K_{b} Z_{\text {set. } .5}^{\mathrm{I}}\right) \text {. }
$$

where $K_{b}$ is the branch coefficient.

When three-phase fault occurs, $K_{b}$ is given by:

$$
K_{b}=1+\frac{\dot{I}_{S}}{\dot{I}_{8}} \text {. }
$$

where $\dot{I}_{S}$ is the output current of $\dot{E}_{S}$, and $\dot{I}_{8}$ is the measured current of relay 8 .

When two-phase fault occurs, branch current at relay 8 only contains positive sequence, so $K_{b}$ can be calculated by:

$$
K_{b}=1+\frac{\dot{I}_{S . b}-I_{S . c}}{\dot{I}_{8 . b}-I_{8 . c}} .
$$

\subsection{Simulation Results}

Faults at the end of Zone I of the relays 3 and 5 are carried out to test the selectivity of Zone II of the relays 2 and 8 , respectively. The amplitude comparison operation equations of relays 2 and 8 are shown in Fig. 6 and Fig. 7, respectively. In both figures, "1" represents the amplitude $\left|Z_{m}-\frac{1}{2} Z_{\text {set }}\right|$, and " 2 " represents the amplitude $\left|\frac{1}{2} Z_{\text {set }}\right|$. From both figures, $\left|Z_{m}-\frac{1}{2} Z_{\text {set }}\right|$ is much bigger than $\left|\frac{1}{2} Z_{\text {set }}\right|$. Therefore, Zone II of relays 2 and 8 will not exceed the protection range of Zone I of the relays 3 and 5, respectively. Selectivity of the two relays can be guaranteed. 


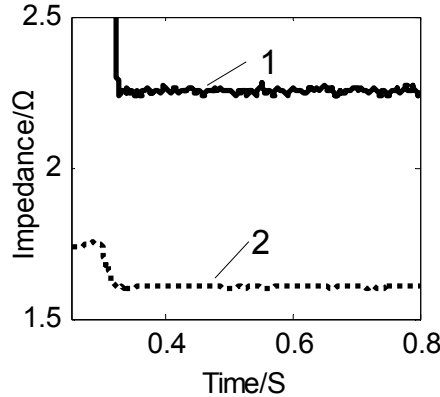

(a) Two phase fault

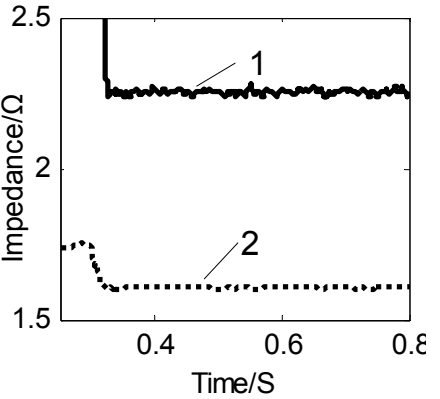

(b)Three phase fault

Fig. 6. Amplitude comparison operation equation of protection 2.

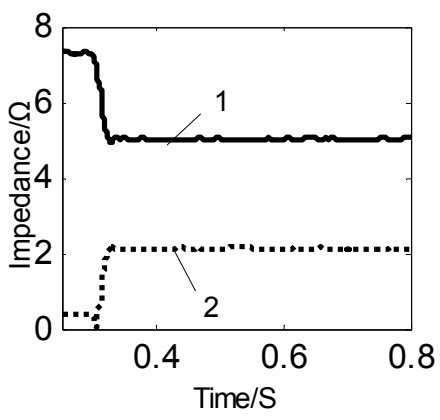

(a)Two phase fault

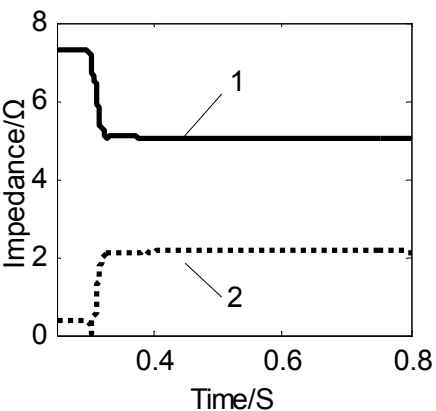

(b)Three phase fault

Fig. 7. Amplitude comparison operation equation of protection 8.

Two-phase and three-phase fault are set on bus C separately to check the sensitivity of relay 2 . Different capacity of the IIDG is considered. Simulation results are shown in Table I.

Table 1 . Sensitivity of Relay 2.

\begin{tabular}{c|c|c|c|c}
\hline \multirow{2}{*}{ S(MVA) } & \multicolumn{2}{|c|}{ Two - phase Fault } & \multicolumn{2}{c}{ Three - phase Fault } \\
\cline { 2 - 5 } & Setting Value & Sensitivity & $\begin{array}{c}\text { Setting } \\
\text { Value }\end{array}$ & $\begin{array}{c}\text { Sensitivit } \\
\mathrm{y}\end{array}$ \\
\hline 10 & 3.8309 & 1.7426 & 3.8561 & 1.7541 \\
\hline 8 & 3.6639 & 1.6666 & 3.6616 & 1.6656 \\
\hline 6 & 3.4799 & 1.583 & 3.4826 & 1.5842 \\
\hline 4 & 3.3031 & 1.5025 & 3.2996 & 1.5009 \\
\hline 2 & 3.1208 & 1.4196 & 3.1182 & 1.4184 \\
\hline 0 & 2.9497 & 1.3418 & 2.9497 & 1.3418 \\
\hline
\end{tabular}

From the simulation results, it can be concluded that zone II of protection 2 has enough sensitivity to clear fault at the end of the protected line.

Table 2. Sensitivity of Relay 8 .

\begin{tabular}{c|c|c|c|c}
\hline \multirow{2}{*}{ S(MVA) } & \multicolumn{2}{|c|}{ Two-phase Fault } & \multicolumn{2}{c}{ Three - phase Fault } \\
\cline { 2 - 5 } & Setting Value & Sensitivity & Setting Value & Sensitivity \\
\hline 10 & 42.0619 & 19.1335 & 42.0811 & 19.1422 \\
\hline 8 & 52.3898 & 23.8315 & 52.4142 & 23.8425 \\
\hline 6 & 69.5983 & 31.6594 & 69.6408 & 31.6787 \\
\hline 4 & 103.9998 & 103.9998 & 104.0834 & 47.3462 \\
\hline 2 & 206.8427 & 94.0902 & 207.2046 & 94.2548 \\
\hline 0 & Exit & Exit & Exit & Exit \\
\hline
\end{tabular}

Then faults on bus A are simulated to verify the sensitivity of protection 8 . Different capacity of the IIDG is considered. Simulation results are shown in Table II.

From the simulation results, it can be concluded that Zone II of relay 8 has enough sensitivity to clear fault at the end of the protected line. 


\section{Conclusions}

This paper presents an adaptive distance protection scheme of distribution system with DG. Firstly, with adaptive distance protection configured at the upstream side of DG, the protected feeders are divided into different zones. Then, on the basis of fault characteristic analysis of the distribution system with DG, the adaptive distance protection scheme is proposed. The results show that this scheme can automatically calculate the settings according to the operation mode and the output power of DG with no need for communication. Simulation results on a $10 \mathrm{kV}$ distribution system have verified scheme has highly acceptable selectivity and sensitivity.

\section{Acknowledgment}

This paper is supported by the Key Project of Science and Technology of State Grid Corporation of China (PD71-13-031).

\section{References}

[1] P. Barker and R. W. de Mello: Determining the impact of distributed generation on power systems: Part 1-Radial power systems, in Proc. IEEE Power Eng. Soc. Summer Power Meeting (2000), p. 1645-1658.

[2] Department of Energy's distributed power program homepage, Information on http://www.eren.doe.gov/der/basics.html.

[3] Portfolio of DG Technologies, Information on http://www.distributed-generation. com/ technologies. Html

[4] H. L. Willis and W. G. Scott: Distributed Power Generation Planning and Evaluation (Marcel Dekker, New York 2000).

[5] Role of distributed generation in competitive energy markets, Information on http://griweb.gastechnology. org/pub/solutions/dg/distgen.pdf.

[6] C. V. Dobariya and S. A. Khaparde: Decoupled power controller for inverter-interfaced distributed generation system, in 2007 IEEE PES General Meeting, p.1-6.

[7] G. Venkataramanan and M. Illindala: Small signal dynamics of inverter interfaced distributed generation in a chain-microgrid, in 2007 IEEE Power Engineering Society General Meeting, p.1-6.

[8] M. A. Anthony: Electric Power System Protection and Coordination (McGraw-Hill, New York 1995).

[9] J. L. Blackburn: Protective Relaying Principles and Applications (Marcel Dekker, New York 1998).

[10]P. M. Anderson: Power System Protection(IEEE Press, New York 1999).

[11]N. Hadjsaid, J. Canard, and F. Dumas: Dispersed generation impact on distribution networks, IEEE Comput. Appl. Power, Vol. 12 (1999), p. 22-28.

[12]Shengwei Li, Yongli Li, Jingliao Sun, Qiang Jin, and Xuguang Li: A novel control algorithm for inverter-based distributed generation in unbalanced three-phase power systems, in 2009 Sustainable Power Generation and Supply Int. Conf., p.1-6.

[13]Tarlochan S. Sidhu, David Sebastian Baltazar, Ricardo Mota Palomino, and Mohindar S. Sachdev: A new approach for calculating zone-2 setting of distance relays and its use in an adaptive protection system, IEEE Trans. Power Delivery, Vol. 19 (2004), p. 70-77.

[14]M.M. Saha, J. Izykowski, E. Rosolowski, M. Bozek: Adaptive line distance protection with compensation for remote end infeed, in 2008 IET Developments in Power System Protection Int. Conf., p.321-326.

[15]Sun Jingliao, Li Yongli, Li Shengwei, and Jin Qiang: Study on adaptive current instantaneous trip protection scheme for distribution network with inverter interfaced DG, Automation of Electric Power Systems, Vol.33 (2009), p. 71-76(in Chinese). 